\title{
The open cluster NGC 6823 and possible triggered star formation associated with SNR G59.5+0.1
}

\author{
Jin-Long $\mathrm{Xu}^{1,2,3}$ and Jun-Jie Wang ${ }^{1,3}$ \\ 1 National Astronomical Observatories, Chinese Academy of Sciences, 100012 Beijing, PR China \\ e-mail: xujl@bao.ac.cn \\ 2 Graduate University of the Chinese Academy of Sciences, 100080 Beijing, PR China \\ 3 NAOC-TU Joint Center for Astrophysics, 850000 Lhasa, PR China
}

Received 20 February 2012 / Accepted 10 April 2012

\section{ABSTRACT}

\begin{abstract}
Aims. We investigate the environment in the vicinity of the supernova remnant (SNR) G59.5+0.1 and identify all the young stellar objects (YSOs) around the SNR, to derive the physical properties, obtain insight into the star-formation history, and determine whether SNR G59.5+0.1 can trigger star formation in this region.

Methods. We perform the submillimeter/millimeter observations in CO lines toward the southeast of SNR G59.5+0.1 with the KOSMA 3 m Telescope. High values of the integrated CO line-intensity ratio $R_{I_{\mathrm{CO}(3-2)} / I_{\mathrm{CO}(2-1)}}$ is identified as one good signature of the SNR-MC (molecular cloud) interacting system. To investigate the impact of SNR G59.5+0.1 on the process of star formation, we use GLIMPSE I catalog to select YSOs (including Class I and Class II sources).

Results. The $\mathrm{CO}$ emission forming an arc-like shape and mid-infrared $8.28 \mu \mathrm{m}$ emission are coincident with SNR G59.5+0.1, which has a total mass of $1.1 \times 10^{4} M_{\odot}$ and fully covers the open cluster NGC 6823. Three molecular clumps are identified in the CO molecular arc, each clump tracing the broad-line wing emission, indicating that there are three outflows in motion. The integrated CO line intensity ratio $\left(R_{I_{\mathrm{CO}(3-2)} / I_{\mathrm{CO}(2-1)}}\right)$ for the whole molecular arc is between 0.48 and 1.57 . The maximum value is 1.57 , which is much higher than previous measurements of individual Galactic MCs. The CO molecular arc has a line-intensity ratio gradient. The SNR G59.5+0.1 is in adiabatic expansion phase. The age of the SNR is $8.6 \times 10^{4} \mathrm{yr}$. Based on the GLIMPSE I catalog, we select 625 YSOs candidates (including 176 Class I sources and 449 Class II sources). The timescales for Class 0 , Class I and Class II sources are $\leq 10^{4} \mathrm{yr}, \sim 10^{5} \mathrm{yr}$, and $\sim 10^{6} \mathrm{yr}$, respectively. The number of YSOs are significantly enhanced in the interacting regions, indicating the presence of some recently formed stars.
\end{abstract}

Key words. ISM: clouds - ISM: individual objects: NGC 6823 - ISM: molecules - stars: formation - ISM: supernova remnants

\section{Introduction}

Massive stars can have a significant impact on the morphology and chemical evolution of the surrounding interstellar medium (ISM) by means of ultraviolet (UV) radiation, stellar winds, and supernova explosions. Stars form from the densest environments in molecular clouds (MCs), which are often clustered together spatially in groups ranging from a few sources to many thousands. When a supernova explodes near MCs, shock generated by a supernova remnant (SNR) may compress some condensed MCs to collapse. On the other hand, the shock can enhance the abundances of the different molecular species with respect to quiescent cloud conditions. Hence, the SNR-MC interaction plays an essential part in the processes of star formation and the evolution of ISM (Reynoso et al. 2001). These associations are established by the detection of $\mathrm{OH} 1720 \mathrm{MHz}$ masers, the broad emission lines, the large ratio of ${ }^{12} \mathrm{CO} J=2-1$ to $J=1-0$ integrated line intensity, $\gamma$-ray emission from MCs toward SNR, as well as the morphological relation between SNR and MCs, and so on (Frail et al. 1996; Green et al. 1997; Hartmann \& Burton 1997; Seta et al. 1998; Wilner et al. 1998; Huang \& Thaddeus 1986; Byun et al. 2006; Hewitt \& Yusef-Zadeh 2009). Jiang et al. (2010) summarized the criteria of the SNR-MCs association. In addition, some results from numerical simulation speculated that star formation can be triggered by the SNR-MC interaction (Melioli et al. 2006; Leao et al. 2009). However, only a few cases of triggered star formation have been identified in the observed results (Junkes et al. 1992; Parons et al. 2009; Xu et al. 2011a).
SNR G59.5+0.1, at a distance of $2.1 \sim 2.3 \mathrm{kpc}$ (Xu et al. 2005; Billot et al. 2010), is situated in the direction of Vulpecula $\mathrm{OB}$ at the position $(l, b)=(59.58,0.12)$. According to Xu et al. (2005), the age of SNR G59.5+0.1 ranges from $10^{3}$ to $10^{4}$ years old. Taylor et al. (1992) described this object as a shell-type SNR with nonthermal spectral index $(\alpha<-0.4)$ and a diameter of $15^{\prime}$. They also mentioned that the SNR is possibly associated with the HII region Sh2-86. Billot et al. (2010) found a circular structure centered at the position of the SNR with a diameter of $15^{\prime}$, which confirms the value found by Taylor et al. (1992). Furthermore, Billot et al. (2010) noted a high density of YSOs lined up along two arcs at the northern and southern rims of SNR G59.5+0.1, suggesting that the interaction of the expanding shell of SNR G59.5+0.1 and the neutral gas of Sh2-86 might have triggered the formation of young stars in the cluster. NGC 6823 is an open cluster located at $\operatorname{RA}(\mathrm{J} 2000)=19^{\mathrm{h}} 43^{\mathrm{m}} 08^{\mathrm{s}} .4, \operatorname{Dec}(\mathrm{J} 2000)=23^{\circ} 18^{\prime} 00^{\prime \prime}$ with a diameter of $\sim 16^{\prime}$ (Kharchenko et al. 2005). Riaz et al. (2012) suggested that NGC 6823 has possibly experienced a recent burst of star formation, which have been caused by the supernova explosion of a massive $\mathrm{O}$ star. However, they suggested that SNR G59.5+0.1 is insufficiently old to trigger any of their identified star formation.

Motivated by the supposed association of SNR G59.5+0.1 with molecular gas and possibly triggered star formation, we performed ${ }^{12} \mathrm{CO} J=2-1,{ }^{12} \mathrm{CO} J=3-2$, and ${ }^{13} \mathrm{CO} J=3-2$ observations toward G59.5+0.1. The observations are described 
in Sect. 2, and the results are presented in Sect. 3. In Sect. 4, we discuss how our data provide evidence of triggered star formation in the interacting region. The conclusions are summarized in Sect. 5.

\section{Observations}

The mapping observations of G59.5+0.1 were performed in ${ }^{12} \mathrm{CO} J=2-1,{ }^{12} \mathrm{CO} J=3-2$, and ${ }^{13} \mathrm{CO} J=2-1$ lines using the KOSMA $3 \mathrm{~m}$ telescope at Gornergrat, Switzerland, in April 2004. The half-power beam widths of the telescope at the observing frequencies of $230.538 \mathrm{GHz}, 345.789 \mathrm{GHz}$, and $220.399 \mathrm{GHz}$ are $130^{\prime \prime}, 80^{\prime \prime}$, and $130^{\prime \prime}$, respectively. The pointing and tracking accuracy was better than $20^{\prime \prime}$. The accuracy of the absolute intensity calibration was better than $15 \%$ (Sun et al. 2008). The DSB receiver noise temperature was about $170 \mathrm{~K}$. The medium and variable resolution acousto optical spectrometers have 1501 and 1601 channels, with total bandwidths of $248 \mathrm{MHz}$ and $544 \mathrm{MHz}$. The channel widths of 165 and $340 \mathrm{kHz}$ correspond to velocity resolutions of 0.21 and $0.29 \mathrm{~km} \mathrm{~s}^{-1}$, respectively. The beam efficiency $B_{\text {eff }}$ is 0.68 at both $230 \mathrm{GHz}$ and $220 \mathrm{GHz}$, and 0.72 at $345 \mathrm{GHz}$. The forward efficiency $F_{\text {eff }}$ is 0.93 for all frequencies. Mapping observations were centered at $\mathrm{RA}(\mathrm{J} 2000)=19^{\mathrm{h}} 43^{\mathrm{m}} 47.85^{\mathrm{s}}, \operatorname{Dec}(\mathrm{J} 2000)=23^{\circ} 21^{\prime} 26.34^{\prime \prime}$ using the on-the-fly mode. The total mapping area is $24^{\prime} \times 24^{\prime}$ in ${ }^{12} \mathrm{CO} J=2-1$ and ${ }^{12} \mathrm{CO} J=3-2$ with a $1^{\prime} \times 1^{\prime}$ grid, while the mapping area in ${ }^{13} \mathrm{CO} J=2-1$ is $10^{\prime} \times 15^{\prime}$.

The data were reduced using the GILDAS/CLASS ${ }^{1}$ package. The correction for the line intensities to the main beam temperature scale was made using the formula $T_{\mathrm{mb}}=\left(F_{\mathrm{eff}} / B_{\mathrm{eff}} \times T_{\mathrm{A}}^{*}\right)$.

\section{Results}

\subsection{Molecular emission}

Figure 1 presents the emission map (color scale) at $8.28 \mu \mathrm{m}$. The emission shows a half-shell morphology, which is associated with SNR G59.5+0.1 and has a diameter of 15'. The integrated intensity map of ${ }^{12} \mathrm{CO} J=2-1$ overlaps with the $8.28 \mu \mathrm{m}$ emission located in the white boxed area of Fig. 1, which covers the open cluster NGC 6823 marked in a green dashed circle (see Fig. 8). The ${ }^{12} \mathrm{CO} J=2-1$ emission coincides well with the $8.28 \mu \mathrm{m}$ emission in the southeast. To analyze in greater detail the morphology of $\mathrm{CO}$ molecular gas, we compiled the integrated intensity map of ${ }^{12} \mathrm{CO} J=3-2$ and ${ }^{13} \mathrm{CO} J=2-1$ (Fig. 2). In Fig. 2, ${ }^{12} \mathrm{CO} J=2-1$ emission is coincident with that from ${ }^{12} \mathrm{CO} J=3-2$ and ${ }^{13} \mathrm{CO} J=2-1$. The ${ }^{12} \mathrm{CO} J=2-1$ and ${ }^{12} \mathrm{CO} J=3-2$ emission appear to show an arc-like structure, while the ${ }^{13} \mathrm{CO} J=2-1$ emission may trace the compact core of molecular gas. We found three cloud clumps in this structure, each clump being designated alphabetically, clumps A, B, and C. Since the mapping area in ${ }^{13} \mathrm{CO} J=2-1$ is smaller (see Sect. 2), we did not detect the ${ }^{13} \mathrm{CO} J=2-1$ emission from clump C. Figure 3 shows ${ }^{12} \mathrm{CO} J=2-1,{ }^{12} \mathrm{CO} J=3-2$, and ${ }^{13} \mathrm{CO} J=2-1$ spectra averaged over clumps A, B, and C, respectively. The line profiles of ${ }^{12} \mathrm{CO} J=2-1$ and ${ }^{12} \mathrm{CO} J=3-2$ for each clump appear to be broadened, the velocity components being mainly located in the velocity interval 18 to $35 \mathrm{~km} \mathrm{~s}^{-1}$. The spectral profile of clump A may have a broad outflow wing and has two weaker components at $23 \mathrm{~km} \mathrm{~s}^{-1}$ and $31 \mathrm{~km} \mathrm{~s}^{-1}$. We performed Gaussian fits to all the spectra. The fitted results

\footnotetext{
${ }^{1}$ http://www.iram.fr/IRAMFR/GILDAS/
}

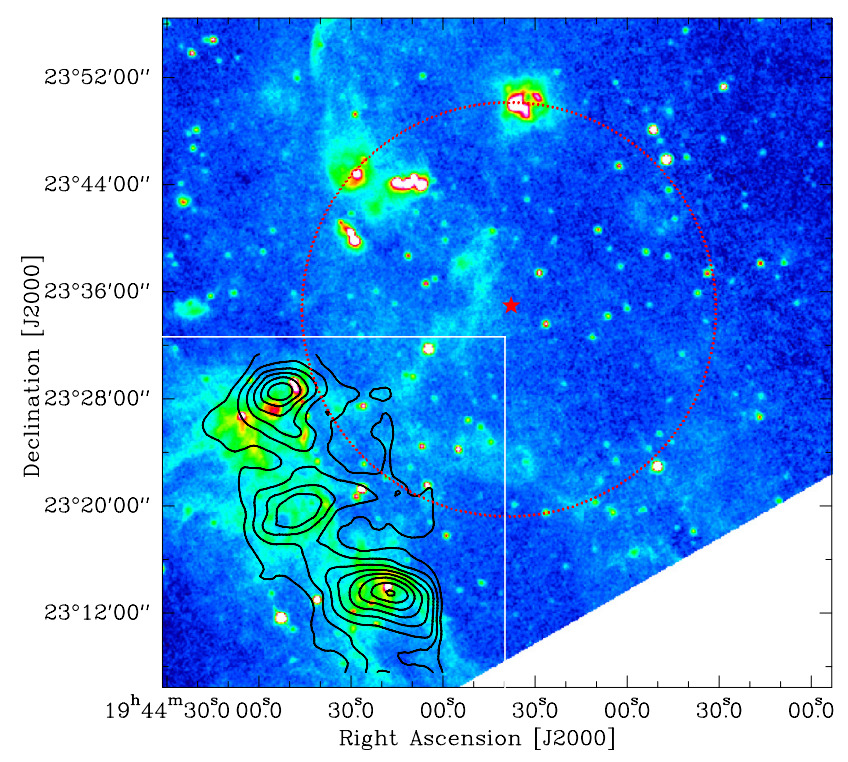

Fig. 1. ${ }^{12} \mathrm{CO} J=2-1$ intensity map (black contours) integrated from 18 to $35 \mathrm{~km} \mathrm{~s}^{-1}$, overlayed on the mid-infrared 8.28- $\mu \mathrm{m}$ MSX emission map (color scale). The contour levels are $30,40, \ldots, 90 \%$ of the peak value $\left(83.9 \mathrm{~K} \mathrm{~km} \mathrm{~s}^{-1}\right)$. The red star represents the center of SNR G59.5+0.1 and its extent is outlined by a red dashed circle. The radius of SNR G59.5+0.1 is 15' (Taylor et al. 1992; Billot et al. 2010). The white box outlines the field seen in Fig. 2.

are summarized in Table 1, from which we derive systemic velocities of $\sim 27.1 \mathrm{~km} \mathrm{~s}^{-1}, \sim 27.5 \mathrm{~km} \mathrm{~s}^{-1}$, and $\sim 28.9 \mathrm{~km} \mathrm{~s}^{-1}$ in clumps A, B, and C, respectively. According to the Galactic rotation model of Fich et al. (1989) together with $R_{\odot}=8.5 \mathrm{kpc}$ and $V_{\odot}=220 \mathrm{~km} \mathrm{~s}^{-1}$, where $V_{\odot}$ is the circular rotation speed of the Galaxy, we obtain a kinematic distance of $2.3 \mathrm{kpc}$ to all clumps, which is roughly consistent with the photometric distance $(2.1 \pm 0.1 \mathrm{kpc})$ of NGC 6823 (Guetter 1992).

Assuming local thermodynamical equilibrium (LTE) and using the ${ }^{12} \mathrm{CO} J=2-1$ line, the column density of the clumps in $\mathrm{cm}^{-2}$ was estimated to be (Garden et al. 1991; Xu et al. 2010)

$N_{\text {clu }}=1.08 \times 10^{13} \frac{\left(T_{\mathrm{ex}}+0.92\right)}{\exp \left(-16.62 / T_{\mathrm{ex}}\right)} \int T_{\mathrm{mb}} \times \frac{\tau \mathrm{d}}{v}[1-\exp (-\tau)]$,

where $\mathrm{d} v$ is the velocity range in $\mathrm{km} \mathrm{s}^{-1}, T_{\mathrm{ex}}$ is the excitation temperature in $\mathrm{K}$, and $\tau$ is the optical depth for the ${ }^{12} \mathrm{CO} J=2-1$ line. We estimated $T_{\mathrm{ex}}$ following the equation $T_{\text {ex }}=11.1 / \ln \left[1+1 /\left(T_{\mathrm{mb}} / 11.1+0.02\right)\right]$, while we calculated $\tau$ according to

$\frac{T_{\mathrm{mb}}\left({ }^{12} \mathrm{CO}\right)}{T_{\mathrm{mb}}\left({ }^{13} \mathrm{CO}\right)} \approx \frac{1-\exp (-\tau)}{1-\exp (-\tau / 89)}$

where $T_{\mathrm{mb}}$ is the corrected main-beam temperature. Here we assume the solar abundance ratio of $\left[{ }^{12} \mathrm{CO}\right] /\left[{ }^{13} \mathrm{CO}\right]=89$ (Lang 1980; Anders \& Grevesse 1989; Garden et al. 1991), and that the ${ }^{12} \mathrm{CO} J=2-1$ emission is optically thick. Additionally, we use the relation $N_{\mathrm{H}_{2}} \approx 10^{4} N_{{ }^{12} \mathrm{CO}}$ (Dickman 1978). If the clumps are approximately spherical in shape, the mean number density $\mathrm{H}_{2}$ is $n\left(\mathrm{H}_{2}\right)=1.62 \times 10^{-19} N_{\mathrm{H}_{2}} / L$, where $L$ is the clump diameter in parsecs (pc). Furthermore, their mass is given by $M_{\mathrm{H}_{2}}=n\left(\mathrm{H}_{2}\right) \frac{1}{6} \pi L^{3} \mu_{\mathrm{g}} m\left(\mathrm{H}_{2}\right)$ (Garden et al. 1991), where 
Table 1. Observed parameters of each line.

\begin{tabular}{|c|c|c|c|c|c|c|c|c|c|}
\hline \multirow[t]{2}{*}{ Name } & \multicolumn{3}{|c|}{${ }^{12} \mathrm{CO} 2-1$} & \multicolumn{3}{|c|}{${ }^{12} \mathrm{CO} 3-2$} & \multicolumn{3}{|c|}{${ }^{13} \mathrm{CO} 2-1$} \\
\hline & $\begin{array}{l}T_{\mathrm{mb}} \\
(\mathrm{K})\end{array}$ & $\begin{array}{c}F W H M \\
\left(\mathrm{~km} \mathrm{~s}^{-1}\right)\end{array}$ & $\begin{array}{c}V_{\mathrm{LSR}} \\
\left(\mathrm{km} \mathrm{s}^{-1}\right)\end{array}$ & $\begin{array}{l}T_{\mathrm{mb}} \\
(\mathrm{K})\end{array}$ & $\begin{array}{c}F W H M \\
\left(\mathrm{~km} \mathrm{~s}^{-1}\right)\end{array}$ & $\begin{array}{c}V_{\mathrm{LSR}} \\
\left(\mathrm{km} \mathrm{s}^{-1}\right)\end{array}$ & $\begin{array}{l}T_{\mathrm{mb}} \\
(\mathrm{K}) \\
\end{array}$ & $\begin{array}{c}F W H M \\
\left(\mathrm{~km} \mathrm{~s}^{-1}\right)\end{array}$ & $\begin{array}{c}V_{\mathrm{LSR}} \\
\left(\mathrm{km} \mathrm{s}^{-1}\right)\end{array}$ \\
\hline Core A & $9.9(0.6)$ & $3.7(0.2)$ & $27.0(0.1)$ & $14.5(0.4)$ & $3.9(0.1)$ & $27.0(0.1)$ & $5.4(0.2)$ & $2.7(0.1)$ & $27.3(0.1)$ \\
\hline Core B & $9.2(0.2)$ & $4.4(0.1)$ & $27.4(0.1)$ & $9.4(0.2)$ & $4.4(0.1)$ & $27.6(0.1)$ & $3.6(0.2)$ & $3.6(0.2)$ & $27.6(0.2)$ \\
\hline Core $\mathrm{C}$ & $13.9(0.3)$ & $4.4(0.1)$ & $28.8(0.1)$ & $18.3(0.3)$ & $4.0(0.1)$ & $29.0(0.1)$ & - & - & - \\
\hline
\end{tabular}

Notes. The position of clump A is $\mathrm{RA}(\mathrm{J} 2000)=19^{\mathrm{h}} 43^{\mathrm{m}} 51.85^{\mathrm{s}}$ and $\operatorname{Dec}(\mathrm{J} 2000)=23^{\circ} 28^{\prime} 26.34^{\prime \prime}$. The position of clump B is at $\mathrm{RA}(\mathrm{J} 2000)=19^{\mathrm{h}} 43^{\mathrm{m}} 47.85^{\mathrm{s}}$ and $\operatorname{Dec}(\mathrm{J} 2000)=23^{\circ} 19^{\prime} 26.34^{\prime \prime}$. The position of clump C is at $\mathrm{RA}(\mathrm{J} 2000)=19^{\mathrm{h}} 43^{\mathrm{m}} 15.85^{\mathrm{s}}$ and $\operatorname{Dec}(\mathrm{J} 2000)=23^{\circ} 13^{\prime} 26.34^{\prime \prime}$.

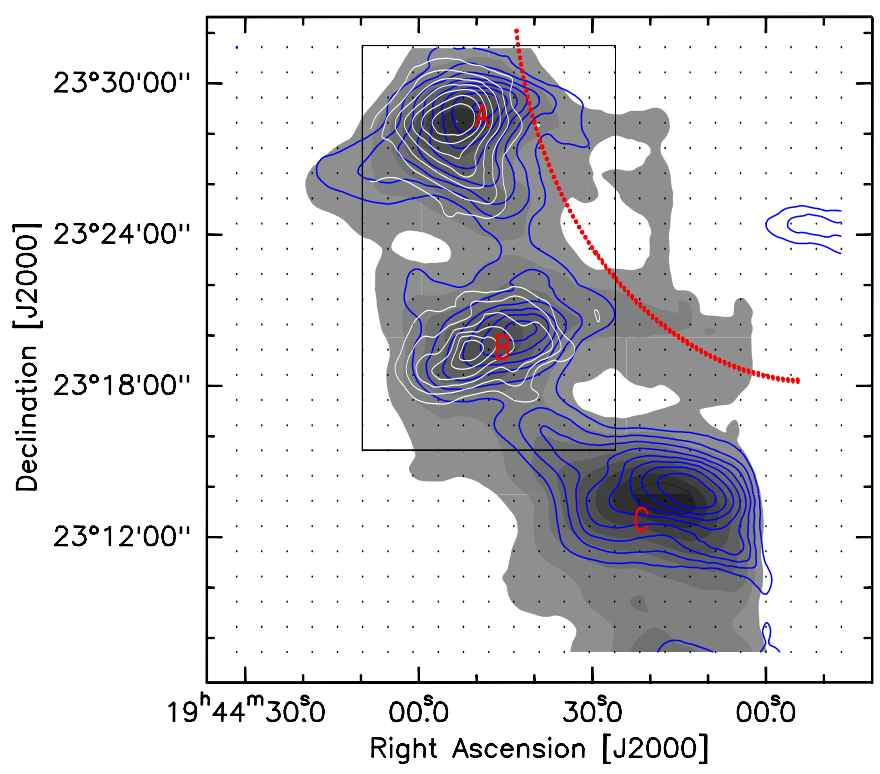

Fig. 2. ${ }^{12} \mathrm{CO} J=2-1$ intensity map (gray scale), superimposed on ${ }^{12} \mathrm{CO} J=3-2$ (blue contours) and ${ }^{13} \mathrm{CO} J=2-1$ (white contours) intensity maps. The contour levels of each $\mathrm{CO}$ molecule are $30,40, \ldots$, $90 \%$ of the peak value. The ${ }^{12} \mathrm{CO} J=3-2$ and ${ }^{13} \mathrm{CO} J=2-1$ peak values are 79.0 and $15.5 \mathrm{~K} \mathrm{~km} \mathrm{~s}^{-1}$, respectively. Letters $\mathrm{A}, \mathrm{B}$, and $\mathrm{C}$ indicate the different $\mathrm{MC}$ clumps. The dot symbols mark the mapping points of ${ }^{12} \mathrm{CO} J=2-1$ and ${ }^{12} \mathrm{CO} J=3-2$, while the black rectangle outlines the mapped field of ${ }^{13} \mathrm{CO} J=2-1$.

$\mu_{\mathrm{g}}=1.36$ is the mean atomic weight of the gas, and $m\left(\mathrm{H}_{2}\right)$ is the mass of a hydrogen molecule. The derived column density, mean number density, and mass of each clump may be incorrect by as much as a factor of three depending on the precise values of the $\left[{ }^{12} \mathrm{CO}\right] /\left[{ }^{13} \mathrm{CO}\right]$ abundance ratio (Garden et al. 1991), which are all listed in Table 2.

To determine the velocity components and confirm the outflow, we made position-velocity (PV) diagrams with the cuts through the peak positions of each clump along the north-south and east-west directions, as shown in Fig. 4, where we can clearly identify three clumps. Each clump shows the bipolar structure marked by the blue and red vertical dashed lines. The blueshifted and redshifted components have obvious velocity gradients, in particular for clumps A and B presented in panels (d) and (e) of Fig. 4. The distributions of redshifted and blueshifted velocity components are an indication of outflow motion. In panel (d), we also identify a velocity component from $21 \mathrm{~km} \mathrm{~s}^{-1}$ to $24 \mathrm{~km} \mathrm{~s}^{-1}$, which does not originate from the component of the outflow but is related to a ${ }^{12} \mathrm{CO} J=2-1$ weaker component peaked at $-23 \mathrm{~km} \mathrm{~s}^{-1}$, as seen from the ${ }^{12} \mathrm{CO} J=2-1$ spectra of clump A in Fig. 3. Using the velocity
Table 2. Physical parameters of the clumps in LTE.

\begin{tabular}{lccccc}
\hline \hline Name & $\begin{array}{c}T_{\text {ex }} \\
\mathrm{K}\end{array}$ & $\begin{array}{c}L \\
\mathrm{pc}\end{array}$ & $\begin{array}{c}N_{\mathrm{H}_{2}} \\
\left(\mathrm{~cm}^{-2}\right)\end{array}$ & $\begin{array}{c}n\left(\mathrm{H}_{2}\right) \\
\left(\mathrm{cm}^{-3}\right)\end{array}$ & $\begin{array}{c}M \\
\left(10^{3} M_{\odot}\right)\end{array}$ \\
\hline Clump A & 15.0 & 5.4 & $1.4 \times 10^{22}$ & $4.2 \times 10^{2}$ & 6.3 \\
Clump B & 14.3 & 4.7 & $0.9 \times 10^{22}$ & $3.3 \times 10^{2}$ & 3.2 \\
Clump C & 19.2 & 6.3 & $2.2 \times 10^{22}$ & $5.6 \times 10^{2}$ & 1.4 \\
\hline
\end{tabular}

ranges obtained from the PV diagram, we made the velocityintegrated intensity maps superimposed on each clump emission map, respectively. The distribution of redshifted and blueshifted velocity components in Fig. 5 provides us with further evidence of the bipolar outflow in each clump.

When a supernova expands into the surrounding molecular clouds, the shocks resulting from the interaction of SNR with MCs can heat the surrounding gases. As the temperature of gases increases, the line opacities decrease as the upper $J$ levels become more populated, and the integrated intensity ratio of ${ }^{12} \mathrm{CO} J=3-2$ to ${ }^{12} \mathrm{CO} J=2-1\left(R_{\left.I_{\mathrm{CO}(3-2)} / I_{\mathrm{CO}(2-1)}\right)}\right)$ can indicate whether there is a the shock (Xu et al. 2011a,b). To obtain the integrated intensity ratio of ${ }^{12} \mathrm{CO} J=3-2$ to ${ }^{12} \mathrm{CO} J=2-1$, the $80^{\prime \prime}$ resolution of ${ }^{12} \mathrm{CO} J=3-2$ data was convolved with an effective beam size of $\sqrt{130^{2}-80^{2}}=102^{\prime \prime}$. We calculated the integrated intensities for ${ }^{12} \mathrm{CO} J=3-2$ line in the same velocity range as for ${ }^{12} \mathrm{CO} J=2-1$ line. The integrated range is from 16 to $36 \mathrm{~km} \mathrm{~s}^{-1}$. Figure 6 shows the distribution of $R_{I_{\mathrm{CO}(3-2)} / I_{\mathrm{CO}(2-1)}}$ (color scale) overlaid with the distribution of ${ }^{12} \mathrm{CO} J=2-1$ line integrated intensity (contours). The red dashed arc represents SNR G59.5+0.1. In Fig. 6, the whole arc-like molecular gas has a ratio value gradient increasing from northeast to southwest, suggesting that the SNR shock is expanding into clump A-C and start to compress each clump. The ratio values for clumps A, B, and $C$ are between 0.48 and 1.57. The maximum value is 1.57 , which is much higher than the typical value $(0.55)$ for MCs in the Galactic disk (Sanders et al. 1993) and value (0.69) for the normal MCs in M 33 (0.69 Wilson et al. 1997), and even higher than the value (0.8) in the starburst galaxy M 82 (Guesten et al. 1993).

\subsection{Infrared emission}

To search for primary tracers of the star-formation activity in the surroundings of G59.5+0.1, we used the GLIMPSE I catalog, which consists of point sources that are detected at least twice in one band. The GLIMPSE I survey observed the Galactic plane $\left(65^{\circ}<|l|<10^{\circ}\right.$ for $\left.|b|<1^{\circ}\right)$ with the four mid-IR bands $(3.6,4.5,5.8$, and $8.0 \mu \mathrm{m})$ of the Infrared Array Camera (IRAC; Fazio et al. 2004) on the Spitzer Space Telescope. From the database, we selected 29469 near-infrared sources within 

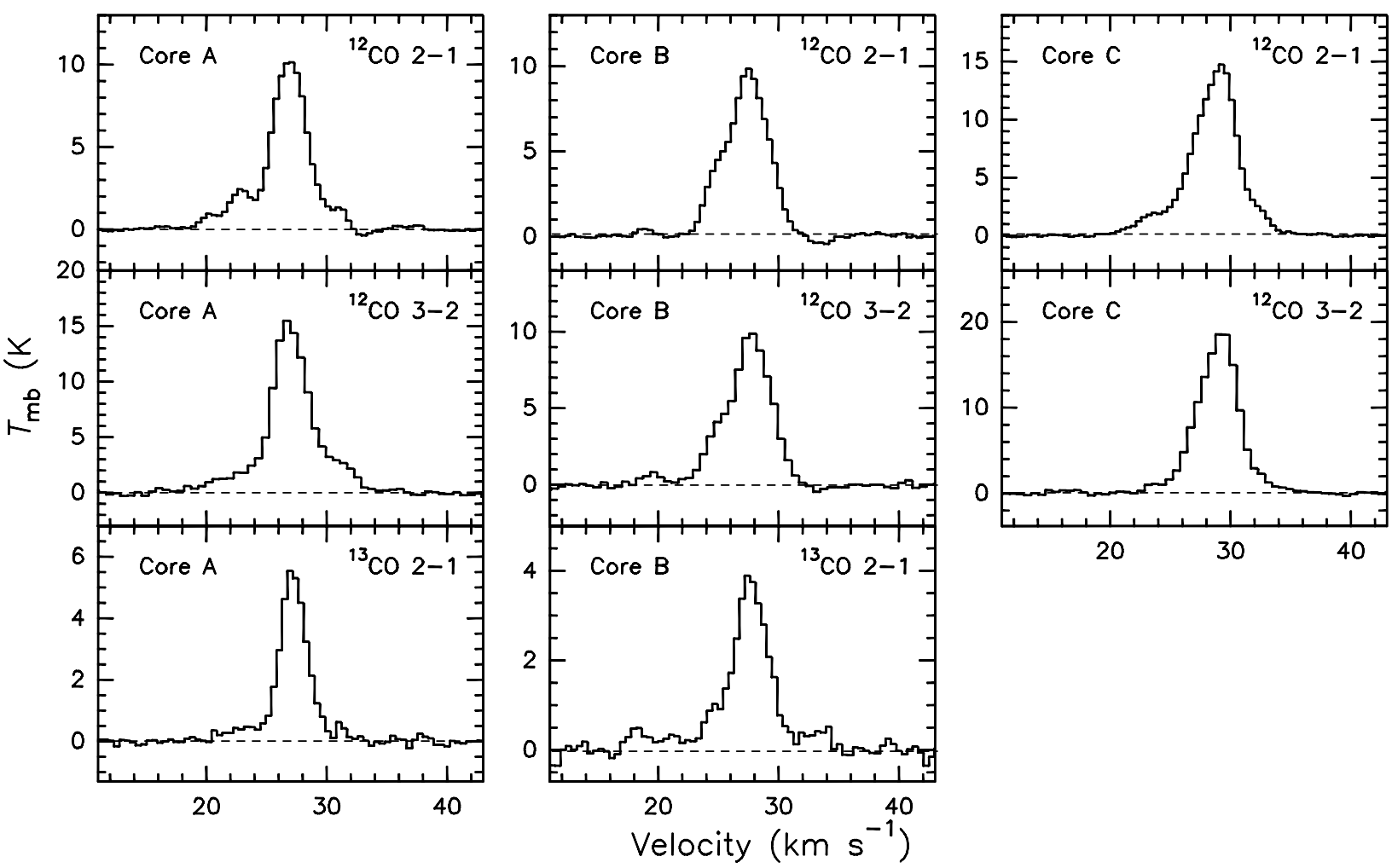

Fig. 3. Average spectra of molecular line ${ }^{12} \mathrm{CO} J=2-1,{ }^{12} \mathrm{CO} J=3-2$, and ${ }^{13} \mathrm{CO} J=2-1$ over each clump. The dashed lines mark the position at the zero intensity.

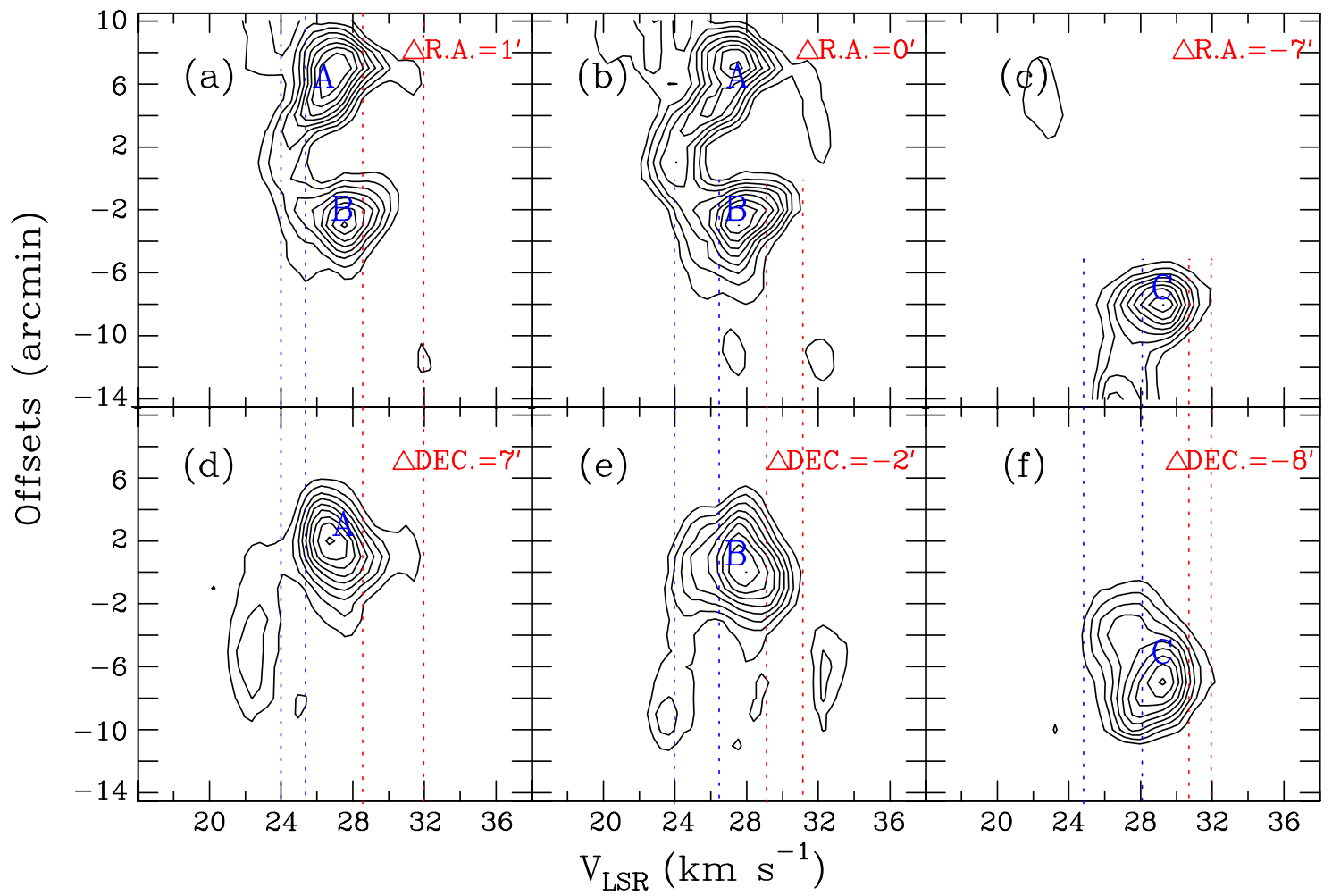

Fig. 4. P-V diagram constructed from CO $J=2-1$ transition for clump A-C. In the a)-c) panels, contour levels are 10, 20, ..., 90\% of the peak value, with a cut through the peak positions of each clump along the north-south direction, and the peak positions are shown in the upper right corner. In the d)-f) panels, contour levels are the same as above panels, with a cut along the east-west direction. The blue and red vertical dashed lines mark the velocity ranges of the blueshifted and redshifted emission for each clump, respectively. The offsets given in each panel are relative to $\operatorname{RA}(\mathrm{J} 2000)=19^{\mathrm{h}} 43^{\mathrm{m}} 47.85^{\mathrm{s}}$ and $\operatorname{Dec}(\mathrm{J} 2000)=23^{\circ} 21^{\prime} 26.34^{\prime \prime}$. 


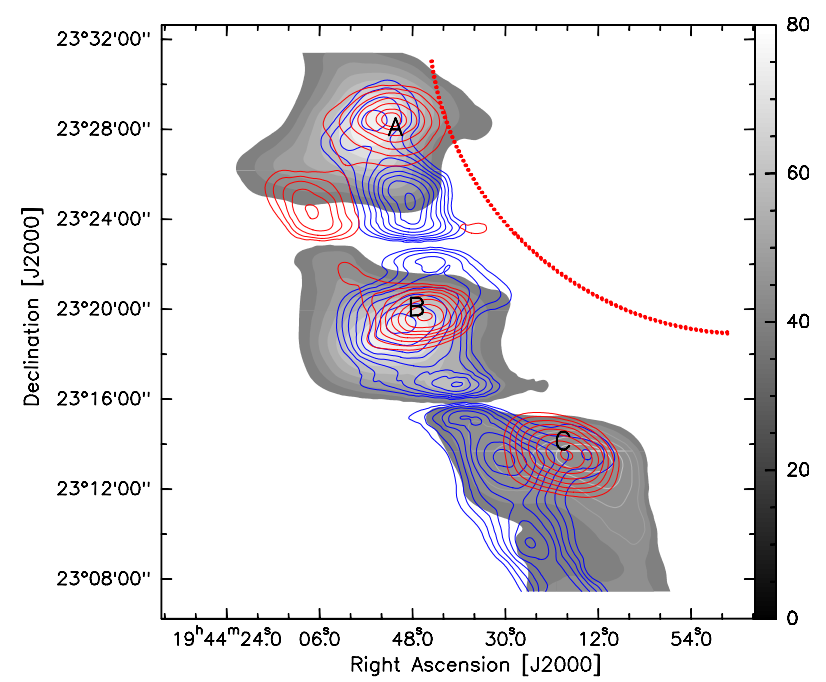

Fig. 5. The velocity-integrated intensity maps of ${ }^{12} \mathrm{CO} J=2-1$ outflows (red and blue contours) overlaid with the ${ }^{12} \mathrm{CO} J=2-1$ emission of each clump (gray scale). The red and blue contour levels are $20,30, \ldots, 100 \%$ of the peak value. The red dashed arc represents SNR G59.5+0.1.

a circle of $24^{\prime}$ in radius centered on $R A=19^{\mathrm{h}} 42^{\mathrm{m}} 50^{\mathrm{s}}(\mathrm{J} 2000)$, Dec $=+23^{\circ} 30^{\prime} 00^{\prime \prime}(\mathrm{J} 2000)$. The size of this region completely covers the extension of G59.5+0.1 and the open cluster NGC 6823. Figure 7 shows the [5.8]-[8.0] versus [3.6]-[4.5] color-color (CC) diagram. The regions in the figure indicate the stellar evolutionary stages based on the criteria of Allen et al. (2004), Parons et al. (2009), and Petriella et al. (2010a). The near-infrared sources are classified into three regions: Class I sources are protostars with circumstellar envelopes (polygon), Class II sources are disk-dominated objects (rectangle), and other sources. Using this criteria, we find 176 Class I sources and 449 Class II sources. Here Class I and Class II sources are chosen to be YSOs.

Figure 8 shows the spatial distribution of both Class I and Class II sources. From Fig. 8, we note that Class I and Class II sources are asymmetrically distributed across the whole selected region, and are mostly concentrated in the north and southeast around SNR G59.5+0.1, which is similar to the results of Billot et al. (2010). Regarding the geometric distribution of Class I and Class II sources, we can plot the map of the star surface density. Because Class I sources is younger than Class II sources and the spatial distribution of Class II sources is similar to that for Class I sources, we only plot the map of the star surface density for Class I sources. This map was obtained by counting all Class I sources with a detection in the $3.6 \mu \mathrm{m}, 4.5 \mu \mathrm{m}, 5.8 \mu \mathrm{m}$, and $8.0 \mu \mathrm{m}$ bands in squares of $4^{\prime} \times 4^{\prime}$, as shown in Fig. 9. From Fig. 9, we can see that there are clear signs of clustering toward the region where SNR G59.5+0.1 is approximately close to the surrounding ISM. The existence of Class I sources may also indicate star formation activity.

\section{Discussion}

\subsection{SNR G59.5+0.1 and MCs}

Owing to the high confusion level in the surroundings of the Sh2-86 structured emission, both Reach et al. (2006) and Billot et al. (2010) did not detect SNR G59.5+0.1. Similarly, we did not detect the SNR in the $8.28 \mu \mathrm{m}$ image, but detected the $8.28 \mu \mathrm{m}$ emissions which presents the half-shell

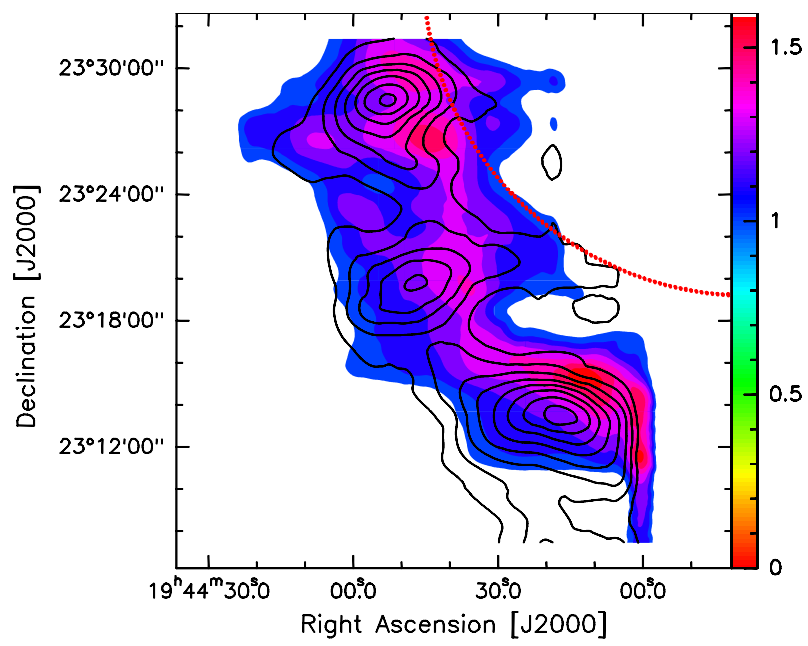

Fig. 6. ${ }^{12} \mathrm{CO} J=2-1$ intensity map are superimposed on the line intensity ratio map (color scale), where the line intensity ratios $\left(R_{I_{\mathrm{CO}(3-2)} / I_{\mathrm{CO}(2-1)}}\right)$ range from 0.48 to 1.57 by 0.16 . The wedge indicates the line-intensity ratio scale.

structure around the SNR. The half-shell emission may be produced by SNR G59.5+0.1 and/or the stellar winds of its progenitor. We performed the submillimeter/millimeter observations in CO lines toward the southeast of SNR G59.5+0.1. The ${ }^{12} \mathrm{CO} J=2-1$ molecular emission is closely coincident with the $8.28 \mu \mathrm{m}$ emission, which covers the whole size of NGC 6823. In addition, the ${ }^{12} \mathrm{CO} J=2-1$ molecular emission has an the arc-like morphology. We identified three clumps in the molecular arc. Three bipolar outflows were detected in these clumps around SNR G59.5+0.1 for the first time. The line profile of ${ }^{12} \mathrm{CO} J=2-1$ in clump A appear to be broadened, which may not be caused only by the outflows, but also by the shock from SNR G59.5+0.1. The maximum value of $R_{I_{\mathrm{CO}(3-2)} / I_{\mathrm{CO}(2-1)}}$ is 1.6 in the molecular arc, which is higher than that in the previous measurement of individual Galactic MCs. A high value of $R_{I_{\mathrm{CO}(3-2)} / I_{\mathrm{CO}(2-1)}}$ has been proposed to be suggested as a signature of the SNR-MC interaction system (Jiang et al. 2010; Xu et al. 2011a). Together, these observations strongly indicate that the MCs are interacting with G59.5+0.1. The SNR G59.5+0.1 appears to have just started to interact with the surrounding MCs, hence the MCs have a non-zero $R_{I_{\mathrm{CO}(3-2)} / I_{\mathrm{CO}(2-1)}}$ gradient along the shock direction. We suggest that the distribution of $R_{I_{\mathrm{CO}(3-2)} / I_{\mathrm{CO}(2-1)}}$ value is a good signature of the SNR-MC interaction system.

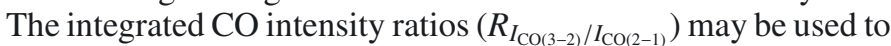
study other triggering mechanisms of star formation, such as the shocks of cloud-cloud collision, HII regions, and galactic density waves. From Table 2, the total mass of the MCs associated with SNR G59.5+0.1 is $1.1 \times 10^{4} M_{\odot}$.

\subsection{Recent star formation in the MCs}

To summarize the results from our investigating of the spatial distribution of YSOs, we can clearly see that the distribution of YSOs (Class I and Class II sources) is clustered around the border of SNR G59.5+0.1. The strongest correlation is found with the CO molecular arc in the southeast where most YSOs may belong to the open cluster NGC 6823. It is unlikely that they are all merely foreground and background stars. It is more likely that these YSOs are physically associated with the interacting regions between G59.5+0.1 and MCs. Riaz et al. (2012) suggested that NGC 6823 have possibly experienced a recent burst 


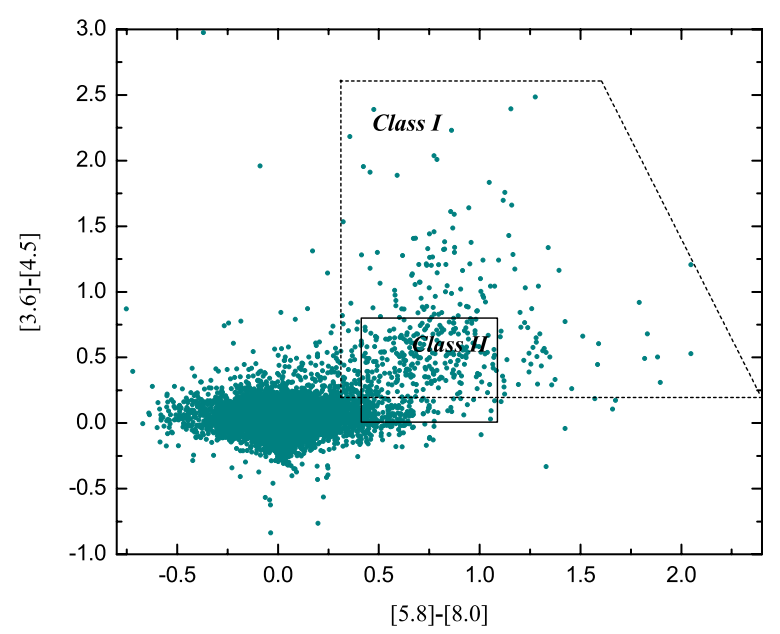

Fig. 7. GLIMPSE color-color diagram [5.8]-[8.0] versus [3.6]-[4.5] for sources. The regions indicate the stellar evolutionary stage as defined by Allen et al. (2004). Class I sources are protostars with circumstellar envelopes and Class II are disk-dominated objects.

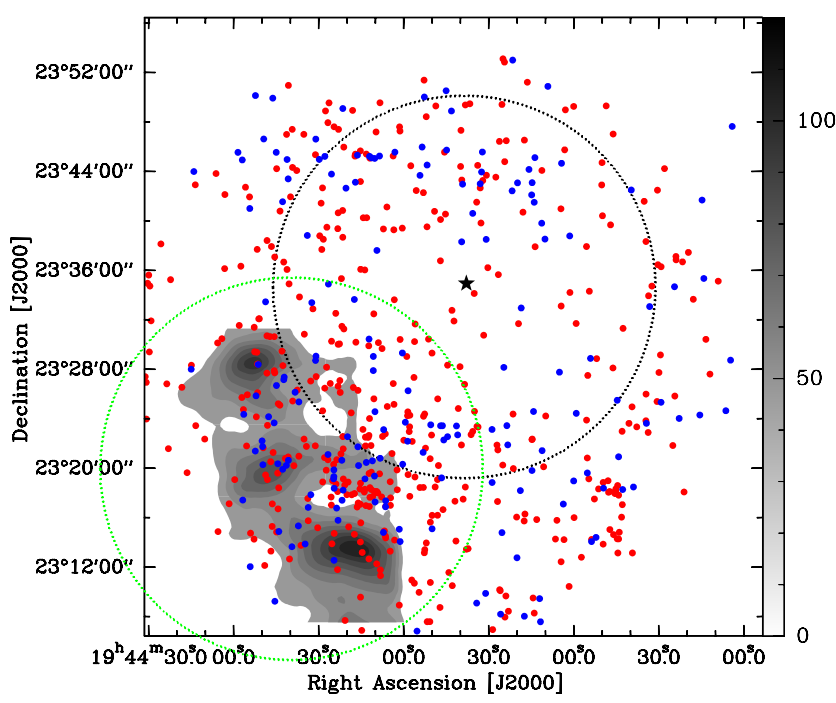

Fig. 8. Positions of Class I and Class II sources relative to the ${ }^{12} \mathrm{CO} J=$ 2-1 MCs (grey scale) around SNR G59.5+0.1. The Class I sources are labeled as the blue dots, and the Class II sources as the red dots. The black and green dashed circles represent SNR G59.5+0.1 and the open cluster NGC 6823, respectively.

of star formation, which may be caused by a supernova explosion of massive $\mathrm{O}$ star. Also, these YSOs distribution is clustered together and shows a shell-like structure around G59.5+0.1. SNR G59.5+0.1 is close approximately to NGC 6823. We suggest that SNR G59.5+0.1 possibly have triggered this YSO formation and star formation in this cluster. Class I sources occur in a period on the order of $\sim 10^{5} \mathrm{yr}$, while the age of Class II sources is $\sim 10^{6}$ yr (André \& Montmerle 1994).

In an inhomogeneous medium, an SNR may undergo different evolutionary stages in different places at the same time. If an SNR is in a radiative expansion phase, the radius of an SNR is $\sim 30$ pc (Heiles 1964). Here the radius of SNR G59.5+0.1 is $\sim 10 \mathrm{pc}$, and the SNR has not yet reached achieved the radiative phase, but remains in adiabatic expansion. The age of G59.5+0.1

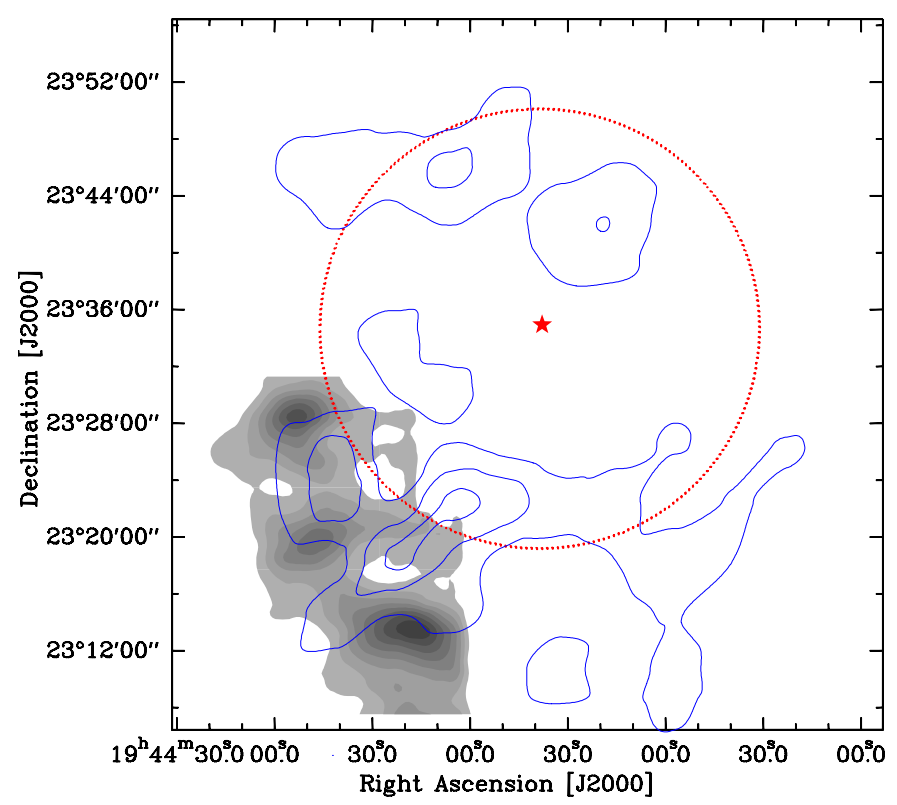

Fig. 9. Stellar-surface density map of Class I candidates are superimposed on ${ }^{12} \mathrm{CO} J=2-1$ MCs (grey scale) around SNR G59.5+0.1. Contours range from two to ten stars $(4 \mathrm{arcmin})^{-2}$ in steps of four stars $(4 \operatorname{arcmin})^{-2} .1 \sigma$ is $0.7(4 \mathrm{arcmin})^{-2}$ (background stars).

given by the Sedov equation (Reynoso \& Mangum 2001) is in units of $10^{4} \mathrm{yr}$

$t_{\mathrm{S}}=\left(R_{\mathrm{S}} / 13.6\right)^{5 / 2}\left(\frac{n_{0}}{E_{51}}\right)^{1 / 2}$,

where $E_{51}$ is the remnant energy, for which we adopt a canonical value of $10^{51} \mathrm{ergs}, R_{\mathrm{S}}$ is the remnant radius, and $\mathrm{n}_{0}$ is the the ambient number density in $\mathrm{cm}^{3}$, which is determined by Table 2. Then we find that the age of G59.5+0.1 is $8.6 \times 10^{4} \mathrm{yr}$, which is shorter than that of these YSOs, which ranges from $10^{5}$ to $10^{6} \mathrm{yr}$. Hence, the formation of the YSOs may not be triggered by the shock of SNR G59.5+0.1. Since SNR G54.4-0.3, SNR G24.7+0.6, and SNR IC443 are not old enough, Junkes et al. (1992), Petriella et al. (2010b), and Xu et al. (2011a) suggested that the YSOs around the remnants may not be triggered by the remnants, but triggered by the stellar winds of the remnant progenitors. SNR G59.5+0.1 is located in the direction of Vulpecula OB at the position $(l, b)=(59.58,0.12)$, hence the spectral type of the SNR G59.5+0.1 progenitor may be either $\mathrm{O}$ or B. In the course of high-mass stellar evolution the stellar winds go through three phases (Dwarkadas 2007), which last for about $4.6 \times 10^{6} \mathrm{yr}$. These periods of stellar winds are sufficient to form YSOs. We conclude that the formation of the YSOs may be triggered by the stellar winds from the high-mass progenitor of G59.5+0.1.

An outflow is strong evidence of this earlier star-forming activity. We detected three outflows around SNR G59.5+0.1. The dynamic timescale of each outflow is given by equation $t=9.78 \times 10^{5} R / V(\mathrm{yr})$, where $R$ in pc is the outflow size defined by the average of the radius of the blueshifted and redshifted lobes, and $V$ in $\mathrm{km} \mathrm{s}^{-1}$ is the maximum flow velocity relative to the cloud systemic velocity. The average dynamical timescales of outflow A-C are $\sim 7.4 \times 10^{3} \mathrm{yr}, \sim 5.3 \times 10^{3} \mathrm{yr}$, and $\sim 5.2 \times 10^{3} \mathrm{yr}$, suggesting that they contain three Class 0 protostars $\left(\leq 10^{4} \mathrm{yr}\right)$ around SNR G59.5+0.1. With a age of $8.6 \times 10^{4} \mathrm{yr}$, SNR G59.5+0.1 may trigger the formation of these 
Class 0 protostars. Observations of higher spatial resolution are needed to further analyze these protostars.

\section{Conclusions}

We have presented the ${ }^{12} \mathrm{CO} J=2-1,{ }^{12} \mathrm{CO} J=3-2$, and ${ }^{13} \mathrm{CO} J=2-1$ molecular and infrared observations towards SNR G59.5+0.1. These results can be summarized as follows:

1. The arc-like morphological association with SNR G59.5+0.1, the broadened emission lines, and the high integrated CO line intensity ratio $\left(R_{\left.I_{\mathrm{CO}(3-2)} / I_{\mathrm{CO}(2-1)}\right)(\sim 1.58) \text { sug- }}\right.$ gest that these cloud clumps are interacting with G59.5+0.1. The age of the SNR is $8.6 \times 10^{4} \mathrm{yr}$. The entire extent of the molecular gas in the southeast has a line-intensity ratio gradient along the direction of shock, implying that shocks have been driven into cloud clumps. We have suggested that high values of $R_{I_{\mathrm{CO}(3-2)} / I_{\mathrm{CO}(2-1)}}$ are reliable probes of the underlying triggering mechanisms of star formation, such as the shocks of cloud-cloud collision, HII regions, and Galactic density waves. The cloud clumps have a total mass of $1.1 \times 10^{4} M_{\odot}$.

2. The selected YSOs (including Class I and Class II sources) are concentrated and grouped around the interacting regions, which appear to be sites of ongoing star formation. Comparing the age of G59.5+0.1 and the timescales of the stellar winds of G59.5+0.1 progenitor with the characteristic star-formation timescales, we conclude that the YSOs may not be triggered by SNR G59.5+0.1, but by the stellar winds of the G59.5+0.1 progenitor.

3 . Three young outflows are detected in the clumps around SNR G59.5+0.1, which may be the first SNR surrounded by more outflows than in all the Galaxy SNRs collectively. Taking into account the age of SNR G59.5+0.1 and Class 0 source, we find that SNR G59.5+0.1 may trigger the formation of these Class 0 sources. It also may provide us a direct evidence for star formation triggered by SNR. The results of the present work confirm the sequential star formation on the basis of the different ages of star formation associated with SNR G59.5+0.1.

Acknowledgements. We would like to thank Dr. Sheng-Li Qin for his help in the data acquirement and discussion. We also thank the anonymous referee for his/her constructive comments and suggestions that greatly improved the content and presentation of this paper. Jin-Long Xu's research is in part supported by a 2011 Ministry of Education doctoral academic prize. This research has been supported by the young Researcher Grant of National Astronomical Observations, Chinese Academy of Sciences.

\section{References}

Allen, L. E., Calvet, N., \& D’Alessio, P. 2004, ApJS, 154, 363 André, P., \& Montmerle, T., 1994, ApJ, 420, 837

Anders, E., \& Grevesse, N. 1989, Geochim. Cosmochim. Acta, 53, 197 Billot, N., Noriega-Crespo, A., Carey, S., et al. 2010, ApJ, 712, 797

Byun, D.-Y., Koo, B.-C., Tatematsu, K., \& Sunada, K. 2006, ApJ, 637, 283

Casoli, F., Combes, F., Dupraz, C., Gerin, M., \& Boulanger, F. 1986, A\&A, 169, 281

Dickman, R. L. 1978, ApJ, 37, 407

Dwarkadas, V. V., 2007, ApJ, 667, 226

Frail, D. A., Goss, W. M., Reynoso, E. M., et al. 1996, AJ, 111, 1651

Fazio, G. G., Hora, J. L., Allen, L. E., et al. 2004, ApJS, 154, 10

Fich, M., Blitz, L., \& Stark, A. A. 1989, ApJ, 342, 272

Garden, R. P., Hayashi, M., Hasegawa, T., et al., 1991, ApJ, 374, 540

Green, D. A., Frail, D. A., Goss, W. M., \& Otrupcek, R. 1997, AJ, 114, 2058

Guesten, R., Serabyn, E., Kasemann, C., et al. 1993, ApJ, 402, 537

Guetter, H. H. 1992, AJ, 103, 179

Hartmann, D., \& Burton, W. B. 1997, Atlas of Galactic Neutral Hydrogen (Cambridge: Cambridge Univ. Press)

Heiles, C. 1964, ApJ, 140, 470

Hewitt, J. W., \& Yusef-Zadeh, F. 2009, ApJ, 694, L16

Huang, Y.-L., \& Thaddeus, P. 1986, ApJ, 309, 804

Jiang, B., Chen, Y., Wang, J. Z. et al. 2010, ApJ, 712, 1147

Junkes, N., Fürst, E., \& Reich, W. 1992, A\&A, 261, 289

Kharchenko, N. V., Piskunov, A. E., Röer, S., Schilbach, E., \& Scholz, R.-D. 2005, A\&A, 438, 1163

Lang, K. R. 1980, Astrophysical Formulae (Berlin: Springer-Verlag), 157

Leao, M. R. M., de Gouveia Dal Pino, E. M., et al. 2009, MNRAS, 394, 157

Melioli, C., de Gouveia Dal Pino, E. M., et al. 2006, MNRAS, 373, 811

Parons, S., Ortega, M. E., rubio, M., \& Dubner, G., 2009, A\&A, 498, 445

Petriella, A., Paron, S., \& Giacani, E. 2010a, A\&A, 513, A44

Petriella, A., Paron, S., \& Giacani, E. 2010b, BAAA, 53, 221

Reach, W. T., Rho, J., Tappe, A., et al. 2006, AJ, 131, 1479

Reynoso, E. M., \& Mangum, J. G. 2001, AJ, 121, 347

Riaz, B., Martín, E. L., Tata R., et al. 2012, MNRAS, 419, 1887

Sanders, D. B., Scoville, N. Z., Tilanus, R. P. J., et al., 1993, in Back to the Galaxy, eds. S. S. Holt, \& F. Verter (New York: AIP), 311

Seta, M., Hasegawa, T., Dame, T. M., et al. 1998, ApJ, 505, 286

Sun, K., Ossenkopf, V., Kramer, C., et al. 2008, A\&A, 489, 207

Taylor, A. R., Wallace, B. J., \& Goss, W. M. 1992, AJ, 103, 931

Wilson, C. D., Walker, C. E., \& Thornley, M. D. 1997, ApJ, 483, 210

Wilner, D. J., Reynolds, S. P., \& Moett, D. A. 1998, AJ, 115, 247

Xu, J. L., \& Wang, J. J. 2010, RAA, 2, 151

Xu, J. L., Wang, J. J., \& Miller, M. 2011a, ApJ, 721, 81

Xu, J. L., Wang, J. J., \& Miller, M. 2011b, RAA, 11, 537

Xu, J. W., Zhang, X. Z., \& Han, J. L. 2005, Chin. J. Astron. Astrophys., 5, 165 